\title{
Adjuvant antibiotic therapy in duodenal ulcers treated with colloidal bismuth subcitrate
}

\author{
T O'Riordan, E Mathai, E Tobin, D McKenna, C Keane, E Sweeney, C O'Morain
}

\begin{abstract}
Persistence of Helicobacter pylori after duodenal ulcer healing is associated with high rates of ulcer relapse. We compared colloidal bismuth subcitrate alone with CBS combined with one of four antibiotic regimens in the treatment of duodenal ulcers. Endoscopy and antral biopsies were performed before treatment and four weeks afterwards. Biopsy specimens were examined for histological evidence of gastritis and by Gram stain and culture for $H$ pylori infection. Altogether 141 patients were allocated to one of five treatment groups. Giving CBS and metronidazole (400 $\mathrm{mg}$ tid for 7 days) with and without amoxycillin $(500 \mathrm{mg}$ tid) achieved higher clearance rates of $\boldsymbol{H}$ pylori than treatment with CBS alone $(\mathbf{p}<0.01)$. These two combinations also achieved higher rates of antral gastritis healing than CBS alone $(p<0.01$ and $p<0.05$ respectively). Susceptibility to metronidazole was tested in 29 isolates before and in seven isolates after treatment with metronidazole by disc diffusion test and minimum inhibitory concentration assay. Twenty seven (93\%) of the isolates were sensitive before treatment while six of seven $(86 \%)$ were resistant afterwards. Four of the six resistant strains had acquired resistance during treatment and one of these had acquired metronidazole resistance despite concomitant treatment with amoxycillin, to which it remained sensitive.

CBS with adjuvant metronidazole at a dose of $400 \mathrm{mg}$ tid for seven days significantly improves the eradication of $\boldsymbol{H}$ pylori compared with CBS alone. Acquired metronidazole resistance, however, seems to be an important cause of failure to eradicate $\boldsymbol{H}$ pylori.
\end{abstract}

There is mounting evidence that Helicobacter pylori is the main cause of antral gastritis. ' Up to $95 \%$ of patients with duodenal ulcer have an associated $H$ pylori positive antral gastritis. ${ }^{2}$ There is a lower incidence of ulcer relapse at one year in patients in whom antral $H$ pylori is eradicated than in those in whom it persists. ${ }^{3+}$ Although colloidal bismuth subcitrate (CBS) and cimetidine have equivalent ulcer healing rates, patients treated with CBS have a lower ulcer relapse rate at one year, and it is postulated that this is due to the well documented activity of CBS against $H$ pylori. ${ }^{3+}$ Nevertheless, CBS alone eradicates $H$ pylori in fewer than $50 \%$ of cases. ${ }^{56}$ In this study patients treated with CBS alone and with CBS in combination with one of four different antibiotic regimens were compared in terms of ulcer healing and antral gastritis and eradication of $H$ pylori.

\section{Methods}

Altogether 141 patients with duodenal ulcer diagnosed at endoscopy, histological evidence of antral gastritis, and microbiological evidence of $H$ pylori infection were included in the study. Patients who had been taking $\mathrm{H}_{2}$ antagonists, bismuth preparations, antibiotics, corticosteroids, and non-steroidal anti-inflammatory drugs during the previous month were excluded. The patients were consecutive and there were no other exclusions.

The patients were allocated to one of five treatment groups. All patients received CBS 120 mg four times a day for four weeks (DeNol swallow tablets-Gist Brocades). The five treatment groups were group 1, CBS alone $(n=43)$; group 2, CBS with amoxycillin (500 mg three times a day for the first week) $(\mathrm{n}=18)$; group 3 , CBS with metronidazole (200 $\mathrm{mg}$ three times a day for the first week) $(n=23)$; group $4, \mathrm{CBS}$ with metronidazole ( $400 \mathrm{mg}$ three times a day for the first week) $(\mathrm{n}=26)$; and group 5, CBS with adjuvant metronidazole $(400 \mathrm{mg}$ three times a day) and amoxycillin (500 $\mathrm{mg}$ three times a day) both for the first week $(n=31)$. As this is a combination of two studies the first 87 patients were allocated by sequential numbers to groups (1), (2), and (3). The remaining 64 patients were allocated by sequential numbers to groups (4) and (5). The endoscopist and histopathologist did not know to which treatment groups the patients belonged. The microbiologist was aware of the treatment groups but this was necessary because of the testing for resistance before and after treatment.

Patients were advised to take CBS half an hour before meals and to take the antibiotics with food. Compliance was monitored using tablet counts. The effectiveness of treatment was assessed by endoscopic healing of duodenal ulcers, healing of histological gastritis, and eradication of $H$ pylori.

Endoscopic examination and antral biopsy from two sites within $2 \mathrm{~cm}$ of the pylorus were performed before treatment and four weeks after the completion of treatment.

One of each pair of biopsy specimens was fixed in $10 \%$ buffered formalin, embedded in paraffin wax, and sectioned. Sections were stained with haematoxylin and eosin and examined for evidence of gastritis. The Warren Marshall classification for gastritis was used:

Grade 0 - normal;

Grade 1 - mild round cell infiltrate;

Grade 2 - massive round cell infiltrate with or without polymorph infiltrate but without epithelial migration or destruction;

Grade 3-polymorph infiltration of the epithelial layers with or without glandular destruction.
Correspondence to: Dr C O'Morain, Departmen of Gastroenterology, Adelaide Dublin 8, Ireland.

Accepted for publication 1 December 1989 
Grades 2 and 3 were regarded as positive for gastritis.

The second biopsy specimen was transported in nutrient broth. A smear of the specimen was Gram stained and the remainder was inoculated onto chocolate agar and incubated at $37^{\circ} \mathrm{C}$ in a humidified aerobic jar flushed with $\mathrm{CO}_{2}$ for up to five days. The growth of small translucent colonies positive for urease (Christensen's urea medium) was taken as positive for $H$ pylori.

Histological and microbiological assessments were carried out without knowledge of clinical details or $H$ pylori status.

The susceptibility to metronidazole of 29 isolates obtained from the $\mathbf{5 7}$ patients in groups 4 and 5 was assessed by disc diffusion technique using $5 \mu \mathrm{g}$ discs and by minimum inhibitory concentration (MIC) determination. The MIC was done on brain-heart infusion agar with $10 \%$ horse blood using a multipoint inoculator. A faintly turbid suspension of isolates in nutrient broth (approximately $10^{6}-10^{7} \mathrm{cfu} / \mathrm{ml}$ ) was used for inoculation. The plates were read after 48 and 72 hours of incubation at $37^{\circ} \mathrm{C}$ in an atmosphere of $\mathrm{CO}_{2}$ and humidity. Amoxycillin sensitivity was tested using amoxycillin discs $(10 \mu \mathrm{g})$ and a zone of inhibition of $5 \mathrm{~cm}$ diameter and above was taken as sensitive. In the disc diffusion test for metronidazole a zone size of $2 \mathrm{~cm}$ diameter and above was considered sensitive.

The results were analysed by $Z$ test.

\section{Results}

The ulcer healing rates with different regimens are shown in Table I. Although ulcer healing was better in groups 4 and 5 , there was no statistically significant difference between the five groups. There was no significant correlation between ulcer healing and $H$ pylori eradication.

Table II summarises the results of $H$ pylori eradication. Group 4 (metronidazole $400 \mathrm{mg}$ ) and group 5 (metronidazole $400 \mathrm{mg}$ and amoxycillin $500 \mathrm{mg}$ ) had significantly higher rates of $H$ pylori eradication than group 1 (on CBS alone) $(\mathrm{p}<0.01)$. Group 2 (amoxycillin $500 \mathrm{mg}$ ) and group 3 (metronidazole $200 \mathrm{mg}$ ) had intermediate eradication rates but did not differ significantly from groups 1,4 , and 5 .

Healing of histological gastritis (Table III) was also significantly better in groups 4 and 5 compared with group $1(\mathrm{p}<0.05, \mathrm{p}<0.05$, and $\mathrm{p}<0.01$ respectively).

The results of sensitivity testing of paired isolates before and after treatment with metronidazole are shown in Table IV. Ninety three per cent of isolates were sensitive to metronidazole before treatment. Six of seven $(86 \%)$ isolates obtained after treatment with metronidazole

TABLE I Duodenal ulcer healing four weeks after completion of treatment in five treatment groups

\begin{tabular}{llll}
\hline Group & No of patients & Healed $(\%)$ & Not healed $(\%)$ \\
\hline 1 CBS & 43 & $28(65)$ & $15(35)$ \\
2 CBS/Amox & 18 & $14(78)$ & $4(22)$ \\
3 CBS/M200 & 23 & $14(61)$ & $9(39)$ \\
4 CBS/M400 & 26 & $22(85)$ & $4(15)$ \\
5 CBS/M400/Amox & 31 & $26(84)$ & $5(16)$ \\
\hline
\end{tabular}

$\mathrm{CBS}=$ colloidal bismuth subcitrate; $M 200=$ metronidazole 200 $\mathrm{mg} ; \mathrm{M} 400=$ metronidazole $400 \mathrm{mg}$; Amox =amoxycillin $500 \mathrm{mg}$.
TABLE II Eradication of $\mathbf{H}$ pylori from the gastric antrum after treatment

$\begin{array}{llll}\hline & & \multicolumn{2}{l}{\text { H pylori }} \\$\cline { 3 - 4 } \text {$\left.Group } & \begin{array}{l}\text { Noof } \\ \text { patients }\end{array} & \text { Eradicated }(\%) & \text { Persistent }(\%) \\ \hline \text { 1 CBS } & 43 & 14(32) & 29(68) \\ 2 \text { CBS/Amox } & 18 & 9(50) & 9(50) \\ 3 \text { CBS/M200 } & 23 & 13(57) & 10(43) \\ 4 \text { CBS/M400 } & 26 & 19(73) & 7(27) \\ 5 \text { CBS/M400/Amox } & 31 & 23(74) & 8(26)\end{array}\right\}$

${ }^{\star} \mathrm{p}<0.05$ (confidence intervals at $1 \%(69 \cdot 8-12 \cdot 2)$ ).

$\star \star \mathrm{p}<0.01$ (confidence intervals at $1 \%(69 \cdot 2-14 \cdot 8)$ )

$\mathrm{CBS}=$ colloidal bismuth subcitrate; $A \operatorname{mox}=$ amoxycillin $500 \mathrm{mg}$;

$M 200=$ metronidazole $200 \mathrm{mg} ; M 400=$ metronidazole $400 \mathrm{mg}$.

were resistant to this drug. Four of these six resistant isolates had acquired resistance during treatment. All isolates were sensitive to amoxycillin before treatment. One isolate which persisted after treatment with metronidazole and amoxycillin had acquired metronidazole resistance but was still sensitive to amoxycillin.

\section{Discussion}

Since the establishment of the link between $H$ pylori eradication and improved ulcer relapse rates efforts have been made to devise ulcer healing treatment regimens that completely eradicate $H$ pylori. Most regimens, such as that used by Coghlan $e t$ al in the study of $H$ pylori and ulcer relapse, have included CBS. ${ }^{3}$ The duration of treatment with CBS in the present study is four weeks as no significant improvement in $H$ pylori clearance seems to be achieved by prolonging treatment. ${ }^{7}$ While CBS $120 \mathrm{mg}$ four times a day is as effective as $240 \mathrm{mg}$ twice a day in healing duodenal ulcer, $H$ pylori eradication is significantly better with the former ${ }^{89}$ Therefore all our patients received CBS $120 \mathrm{mg}$ four times a day.

Although $H$ pylori is sensitive in vitro to a wide range of antibiotics including penicillin, metronidazole, tinidazole, cephalosporins, some quinolones, gentamicin, tetracycline, erythromycin, and rifampicin, the clinical efficacy of most of these is poor. ${ }^{10-12}$ Erythromycin, for example, is not effective in the low $\mathrm{pH}$ environment of the stomach.

In this study the best clearance rates for $H$ pylori were achieved by the two groups that received metronidazole at a dose of $400 \mathrm{mg}$ three times a day for seven days. These groups also had the highest rates of gastritis healing.

The $H$ pylori eradication rates in this study (74\%) are comparable with the results achieved by Borsch et $a l,{ }^{13}$ who, in a pilot study of peptic ulcer patients with $H$ pylori positive gastritis, used a two week combination of bismuth subsalicytate (600 mg three times a day), metro-

TABLE III Effect of treatment on healing of antral gastritis

$\left.\begin{array}{llll}\hline \text { Group } & \text { No of patients } & \text { Healed }(\%) & \text { Persistent }(\%) \\ \hline \text { 1 CBS } & 43 & 17(40) & 26(60) \\ 2 \text { CBS/Amox } & 18 & 11(61) & 7(39) \\ 3 \text { CBS/M200 } & 23 & 12(52) & 11(48) \\ 4 \text { CBS/M400 } & 26 & 18(69) & 8(31) \\ 5 \text { CBS/M400/Amox } & 31 & 22(71) & 9(29)\end{array}\right]$

${ }^{\star} \mathrm{p}<0.05$ (confidence intervals at $5 \%(31 \cdot 4-26 \cdot 6)$

${ }^{\star}{ }^{\star} \mathrm{p}<0.05$ (confidence intervals at $1 \%(34 \cdot 1-27 \cdot 8)$ )

$\mathrm{CBS}=$ colloidal bismuth subcitrate; Amox = amoxycillin; $\mathrm{M} 200=$ metronidazole $200 \mathrm{mg}$; $M 400=$ metronidazole $400 \mathrm{mg}$. 
TABLE IV Development of metronidazole resistance in $\mathrm{H}$ pylori with treatment

\begin{tabular}{llll}
\hline Treatment & No tested & Sensitive $(\%)$ & Resistant (\%) \\
\hline Before & 29 & $27(93)$ & $2(7)$ \\
After & 7 & $1(14)$ & $6(86)$ \\
\hline
\end{tabular}

nidazole (500 $\mathrm{mg}$ three times a day), and amoxycillin ( $500 \mathrm{mg}$ three times a day) and achieved $90 \%$ eradication rate four weeks after completion of treatment. Some of the patients were given $\mathrm{H}_{2}$ antagonists with the above regimen. No antibiotic toxicity was reported.

Marshall et $a l^{4}$ improved the $H$ pylori eradication rate in duodenal ulcer patients from $27 \%$ to $70 \%$ by adding tinidazole ( $500 \mathrm{mg}$ twice a day for 10 days) to an eight week course of CBS (120 mg four times a day). Minor toxic effects were more common in patients treated with tinidazole.

A study by Borody et $a l^{14}$ (including both duodenal ulcer and non-ulcer dyspeptic patients) used a four week combination of CBS (120 mg four times a day), tetracycline ( $500 \mathrm{mg}$ four times a day), and metronidazole ( $200 \mathrm{mg}$ four times a day) for two weeks. Toxic effects seem to have been more common with this regimen than with the other above mentioned regimens. The eradication rate of $H$ pylori eight weeks after treatment was $94 \%$. A follow up of 21 duodenal ulcer patients who were $H$ pylori negative at the eight week stage showed that only three (14\%) had become $H$ pylori positive and all of these positive patients had an ulcer relapse. Interpretations of the findings of this study are complicated by the fact that some of the patients received amoxycillin rather than tetracycline.

Antibiotics alone or in combination with $\mathrm{H}_{2}$ antagonists have a poor record in eradicating $H$ pylori gastritis despite impressive in vitro sensitivity. ${ }^{14}$ is This failure seems to be due to the development of antibiotic resistance during treatment. Bayerdorffer $e t$ al found that virtually all strains of $H$ pylori treated with a combination of ofloxacin and bismuth developed resistance to ofloxacin during treatment. ${ }^{16}$ Goodwin et al found that patients treated with a combination of cimetidine and tinidazole had very high rates of acquired tinidazole resistance. In contrast the development of tinidazole resistance was almost abolished by concomitant treatment with CBS. ${ }^{17}$ In the present study of seven patients with persistent $H$ pylori infection, two were infected with resistant strains and four acquired resistance to metronidazole during treatment. Acquisition of resistance may be a major factor in determining $H$ pylori eradication and seems to be more common than pretreatment resistance. The addition of CBS to metronidazole did not prevent the development of resistance. We assume, but cannot prove, that the persistence of $H$ pylori in these patients is due to resistance rather than early infection, as this concurs with experience of other studies. ${ }^{+16}$

All isolates in our study were sensitive to amoxycillin before treatment. Amoxycillin resistance did not develop in the one strain which was tested after treatment with combined amoxycillin and metronidazole and in which metronidazole resistance had developed. A possible explanation for this occurrence is suggested by Hollingsworth et al, ${ }^{18}$ who found that metronidazole was secreted in gastric mucus whereas amoxycillin was not and who postulated that some antibiotics may be active only topically resulting in high but transient clearance of $H$ pylori with the organism persisting in the gastric pits. It seems that the best clinical results for eradication of $H$ pylori are obtained by regimens that include imidazoles, despite in vitro studies showing that amoxycillin and other agents have higher sensitivity rates. " 12 It is also worth noting that metronidazole was found to have equivalent ulcer healing to cimetidine in a study that did not assess $H$ pylori status. ${ }^{19}$

Eradication of $H$ pylori is increasingly regarded as the single most important prognostic factor for duodenal ulcer relapse. ${ }^{5}$ Our results justify the use of adjuvant metronidazole at a dose of $400 \mathrm{mg}$ three times a day for seven days in duodenal ulcer patients treated with CBS. It produced a $73 \%$ clearance with no appreciable toxicity. We were unable to show a significant benefit from the addition of amoxycillin to metronidazole at this dose. Because of the size of the groups, a small additional benefit from amoxycillin cannot be excluded on statistical grounds. Furthermore, we have shown the development of metronidazole resistance not only in the presence of CBS but also in the presence of amoxycillin to which the isolate was sensitive indicating that adjuvant amoxycillin may not prevent metronidazole resistance. The increasing awareness of $H$ pylori's ability to develop resistance to imidazoles suggests that this may be the main barrier to $H$ pylori clearance. It is reasonable to look for another antibacterial agent to counter this resistance. To date the evidence from this and other studies has yet to show conclusively that amoxycillin should be used with CBS and metronidazole to achieve total clearance of $H$ pylori.

1 Marshall BJ. Unidentified curved bacilli on gastric epithelium in active chronic gastritis. Lancet 1983; i: 1273-5.

2 Dooley CP, Cohan H. The clinical significance of Campylobacter pylori. Ann Intern Med 1988; 108: 70-9.

3 Coghlan JG, Gilligan D, Humphreys H, et al. Campylobacter pylori and recurrence of duodenal ulcers - a 12 month follow up study. Lancet 1987; ii: 1109-11.

4 Marshall B, Goodwin CS, Warren JR, et al. Prospective double blind trial of duodenal ulcer relapse after eradication of Campylobacter pylori. Lancet 1988; ii: 1437-42.

5 Goodwin CS. Duodenal ulcer, Campylobacter pylori and the 'leaking roof' concept. Lancet 1988; ii: 1467-9.

6 Humphreys H, Bourke S, Dooley C, et al. Effect of treatment on Campylobacter pylori in peptic disease. A randomised prospective trial. Gut 1988; 29: 279-83.

7 Lambert JR, Borromeo M, Eaves ER, Hanskey J, Korman M Efficacy of different dosage regimes of bismuth in eradicating Campylobacter pylori. Gastroenterology 1988; 94: A248.

8 Coghlan JG, Gilligan D, Humphries H, et al. Efficacy of different dosage regimens in duodenal ulcer healing and eradication of Campylobacter pylori [Abstract]. Gut 1987; 28: A1409.

9 Hollanders D. Twice daily tripotassium dicitrato bismuthate in the treatment of duodenal ulceration. Postgrad Med $\mathcal{J}$ 1986; 62: 19-21.

10 Marshall BJ, McGechie DB, Rogers PA, Glancy RJ. Pyloric campylobacter infection and gastroduodenal disease. Med $\mathscr{J}$ Aust 1985; 142: 439-44.

11 Van Caekenberg D, Breyssens J. In vitro synergistic activity between bismuth subcitrate and various antimicrobial bents against Campylobacter pyloridis (C. pylori). Antimicrob Agents Chemother 1987; 31: 1429-30.

12 McNulty CAM, Dent JC, Ford GA, Wilkinson SP. Inhibitory antimicrobial concentrations against $C$. pylori in gastric antimicrobial concentrations against $C$. pylon in
mucosa. F Antimicrob Chemother 1988; 22: 729-38.

13 Borsch A, Mai U, Opperkuch W. Oral triple therapy (OTT) may effectively eradicate $C$. pylori in man: A pilot study. may effectively eradicate $C$. pylor

14 Borody T, Cole P, Noonan S, et al. Long term Campylobacter pylori recurrence post-eradication. Gastroenterology 1988; 94 : A43. 
15 Oderda G, Dellolio D, Morra I, Ansaldi N. Campylobacter pylori gastritis long term results of treatment with amoxycillin. Arch Dis Child 1989; 64: 326-9.

16 Bayerdorffer E, Simon TH, Bastlein $\mathrm{CH}$, Ottenjann $\mathrm{R}$ Kasper G. Bismuth/ofloxacin combination for duodena ulcer. Lancet 1987; ii: 1467-8.

17 Goodwin C, Marshall BJ, Blincow ED, Wilson DH, Blackbourn S, Phillips M. Prevention of nitroimidazole resistance in Campylobacter pylori by co-administration of colloidal bismuth subcitrate: clinical and in vitro studies. f Clin Pathol 1987; 41: 207-10.

18 Hollingsworth J, Goldie J, Silletti Li, Richardson H, Hunt RH. Gastric secretion of antibiotics used for Campylobacter RH. Gastric secretion of antibiotics used for Campylobacter
pylorid [Abstract]. Gut 1987; 28: A 1409. 19 Quintero Diaz M, Sotto Eschobar A. Metronidazole versus cimetidine in treatment of gastroduodenal disease. Lancet 1986; i: 907. 